\title{
植被演替过程中种群格局动态的分形分析
}

\author{
王本洋 余世孝* 王永繁 \\ (中山大学生命科学学院生态学系/有害生物控制与资源利用国家重点实验室, 广州 510275)
}

\begin{abstract}
摘 要 该文根据 空间代替时间” 的原则和对马尾松 (Pinus massoniana) 种群的重要值-平均胸径的变动趋势的分 析，确定 5 个森林群落为广东省黑石顶自然保护区森林群落演替的一个时间序列; 并在此基础上,运用分形理论的 计盒维数和信息维数, 对演替过程中马尾松种群空间格局的动态进行了分形分析。结果表明, 马尾松种群的计盒 维数和信息维数 2 个参数值均呈递减趋势, 与其个体数、重要值变动趋势一致。在群落的演替过程中,马尾松种群 的空间占据能力不断下降, 种群呈衰退趋势。随其在群落中的优势地位逐渐被其它种群取代, 群落将由以马尾松 占绝对优势的单优群落演替为多优常绿阔叶林群落。分析结果同时表明, 分形分析是群落演替过程中种群空间格 局动态研究的有效方法 , 分形维数则能反映种群格局的尺度变化规律。
\end{abstract}

关键词 森林群落 演替 格局动态 黑石顶 计盒维数 信息维数

\section{FRACTAL ANALYSIS OF THE DYNAMICS OF POPULATION PATTERNS DURING VEGETATION SUCCESSION}

\author{
WANG Ben-Yang YU Shi-Xiao* and WANG Yong-Fan \\ ( Department of Ecology , School of Life Sciences/State Key Laboratory of Biocontrol, Sun Yat-Sen University , Guangzhou 510275 , China)
}

\begin{abstract}
Background and Aims The dynamics and fractal characteristics of population patterns during vegetation succession are rarely explored. Scale, pattern and process of ecological succession are three intertwined concepts in modern ecology. Succession research will inevitably involve scale and pattern analyses. Fractal theory can be employed as an effective tool for synergic analysis of scale , pattern and successional processes. There are few reports on pattern dynamics of Pinus massoniana, a species that declines during succession in southern China. Five forest communities were selected in Heishiding Nature Reserve, Guangdong Province for a case study. Our objectives were to determine 1) whether results obtained by employing fractal analysis are consistent with those from traditional research where space is substituted for time and 2) the pattern dynamics of $P$. massoniana and the ecological meaning of the change of fractal characteristics in succession.
\end{abstract}

Methods For the traditional space-for-time succession approach, we analyzed importance value (IV) and mean diameter at breast height $(D B H)$ of $P$. massoniana , and the selected forest communities were treated as a time series of five successional stages. The box-counting dimension (Dbox) and information dimension (Dinfo) from fractal theory were employed to analyze the pattern dynamics of $P$. massoniana .

Key Results Dbox and Dinfo of $P$. massoniana decreased during succession, in accordance with number of individuals and $I V$, and the traditional method of substituting space for time was applicable in this study. This demonstrated deteriorating regeneration conditions and decreasing ability of spatial occupation of P. massoniana with succession - that $P$. massoniana had a declining population. With its loss of dominance, the community would turn from $P$. massoniana dominance into a multi-population dominated evergreen broadleaved forest community. The results of fractal analysis were not only consistent with those of the trend analysis of $I V$ and mean $D B H$, but also yielded more ecological information about scale-related spatial pattern of the population , its degree of spatial occupation and its role in community and regeneration conditions.

Conclusions This study indicated that fractal analysis is an effective approach to study dynamic pattern analysis during forest succession, and the results were more reliable than those obtained by employing only $I V$ and mean $D B H$ in a traditional space-for-time approach.

Key words Forest community , Succession , Pattern dynamics , Heishiding Nature Reserve , Box-counting dimension , Information dimension 
马尾松( Pinus massoniana) 耐干旱、痊土, 生长迅 速, 适应性强, 是我国南方主要造林树种, 也是南亚 热带植被恢复过程中重要的先锋树种之一。已有研 究结果普遍认为, 我国南亚热带地区存在着由马尾 松林向常绿榈叶林演替的趋势 (王伯荪和马曼杰, 1982）,从演替过程来看, 马尾松属于南亚热带森林 群落演替过程中的衰退种群(备启杰等, 2000) , 光照 对其种子萌发、幼苗及成树生长的影响被认为是该 种群衰退的主要原因(丁圣彦和宋永昌,1998)。马 尾松群落演替过程中的变化特征, 也有一些研究报 道(方炜和彭少麟，1995;杨清培等，2001)，但均未涉 及马尾松种群空间格局的动态变化及其分形特征。

现代观点认为，演替不仅包含生态系统在时间 序列上的替代过程, 而且也包含生态系统在空间上 的动态演变, 演替系列的格局、尺度、方向等都是演 替的重要空间属性(江洪等，2003），生态演替过程与 演替系列的格局、尺度之间有着重要的关系。尺度、 格局和过程已成为生态学问题中互相联系、不可分 割的 3 个重要概念, 对演替过程的研究不可避免地 要涉及到对尺度和格局的相关研究。王本洋和余世 孝(2005)提出扩展最近邻体分析方法并对种群格局 进行了多尺度分析。分形理论为尺度和格局分析提 供了更为简洁有效的手段, 但应用于群落演替过程 中分析种群格局动态的研究还未见报道。

分析群落演替过程中马尾松种群空间格局的动 态及其分形特征, 不仅有助于深刻认识该种群在群 落中的地位、作用及其变动趋势, 深入理解南亚热带 森林群落演替动态及其种群更替规律, 还可为次生 林林分改造及植被恢复、生物多样性保护与维持等 提供相关的理论支持。

本文以广东省黑石顶自然保护区 5 个森林群落 的演替过程为研究对象，根据 空间代替时间” 的原 则,利用马尾松种群的重要值-平均胸径的变动趋 势, 确定群落演替的时间系列。在此基础上, 应用计 盒维数和信息维数两种分形维数, 探讨本研究中使 用 空间代替时间” 原则的有效性, 分析群落演替过 程中马尾松种群空间分布格局的动态变化及其分形 特征。

\section{1 研究地与样地设置}

黑石顶自然保护区地处南亚热带北缘，位于广 东省封开县境内 , $111^{\circ} 53^{\prime} \mathrm{E}, 23^{\circ} 27^{\prime} \mathrm{N}$, 面积 4000 多 公顷。大部地区海拔约 $150 \sim 700 \mathrm{~m}$, 主峰黑石顶海 拔 $928 \mathrm{~m}$; 地势东南高而西北低,属低山山地地貌。
其位于北回归线附近，雨量丰富，年降雨量 1743.88 $\mathrm{mm}$, 湿度较大, 年平均相对湿度在 $80 \%$ 以上, 属南亚 热带季风气候(余世孝等,2000)，为多种热带、亚热 带生物的生长提供了极为优越的条件。

按各样地所处环境特征尽量相似的原则，在黑 石顶自然保护区的茅坪、石门塘西北、石门塘东北、 莫乃坑及独田等地按典型取样法各设置一块面积为 $60 \mathrm{~m} \times 60 \mathrm{~m}$ 的样地, 共划分为 36 个 $10 \mathrm{~m} \times 10 \mathrm{~m}$ 的 样方。在各样地内进行编号并进行群落学调查, 调 查对象为胸径大于 $1 \mathrm{~cm}$ 的乔灌木树种, 记录其种 名、树高、胸径和样方内坐标等数量指标, 同时记录 各样地坡向、坡度、海拔高度等环境数据。

\section{2 分析方法}

地理信息系统 ( Geographic information system , GIS)技术以其强大的空间数据处理能力成为开发专 业应用系统的理想技术平台 (王本洋和余世孝， 2003) 其应用领域从景观分析逐渐渗透到种群和群 落分析中 (余世孝等,1998,2001 ;戴小华等,2003; 王 本洋和余世孝, 2005), 是跨层次生态学研究的重要 技术工具。

\section{1 重要值}

利用基于 GIS 软件 ArcView 技术平台的自编扩 展模块对样地数据进行处理, 以获取各样地的群落 学特征数据。其中, 计算相对频度时使用的小样方 大小为 $10 \mathrm{~m}$, 显著度为胸高断面积之和, 计算乔灌 层树种重要值 (Importance value, $I V$ ) 为 :

$I V=($ 相对多度 + 相对频度 + 相对显著度 $)$

\section{2 分形维数}

利用基于 GIS 软件 ArcView 技术平台的自编扩 展模块对样地数据进行处理, 以获取各样地中指定 树种的计盒维数和信息维数。

\subsection{1 计盒维数}

计盒维数的计算公式为：

$$
D_{b}=\lim _{\varepsilon \rightarrow 0} \frac{\log (N(\varepsilon))}{-\log (\varepsilon)}
$$

式中 : $\varepsilon$ 为对样地进行栅格化处理时的网格边长, $N$ $(\varepsilon)$ 为对应的非空格子数。本文把样地边长由 2 等 分 (对应网格边长为 $30 \mathrm{~m}$ ) 到 15 等分 (对应网格边长 为 $4 \mathrm{~m})$ 共划分 14 次。利用所得的 $\varepsilon-N(\varepsilon)$ 数据， 在双对数坐标下进行直线 (或分段直线) 拟合, 所得 拟合直线斜率的绝对值即为计盒维数的估计值 (Sugihara \& May ,1990)。

\section{2 .2 信息维数}


种群格局信息维数 ( Information dimension) 能够 刻划格局强度的尺度变化程度, 揭示种群个体分布 的非均匀性状况, 其数值差异可反映更新状况的差 异(马克明和祖元刚，2000a)。

在计盒维数的基础上,信息维数进一步考虑了 每一非空格子中的个体数, 并通过信息量公式给出 尺度及其对应信息量间的幕律关系。其计算公式 为:

$$
D_{i}=\lim _{\varepsilon \rightarrow 0} \frac{I(\varepsilon)}{-\ln (\varepsilon)}
$$

其中, $I(\varepsilon)$ 为总的信息量, 计算公式为 $I(\varepsilon)=\sum I_{i}$ 。 信息量 $I_{i}=-P_{i} \ln P_{i}, P_{i}=N_{i} / N\left(N_{i}\right.$ 为非空格子内 的个体数, $N$ 为样地内总的个体数)。利用所得的 $\varepsilon$ $-I(\varepsilon)$ 数据, 在双对数坐标下进行直线(或分段直 线)拟合，所得拟合直线斜率的绝对值即为信息维数 的估计值(马克明和祖元刚，2000a)。

\section{3 结果与分析}

\section{1 重要值-平均胸径分析}

黑石顶 5 个森林群落的优势种见表 1 (只列出

表 1 黑石顶自然保护区 5 个森林群落的优势种

Table 1 Dominant species within the five forest communities in Heishiding Nature Reserve , Guangdong Province

\begin{tabular}{|c|c|c|c|c|c|c|c|c|}
\hline $\begin{array}{c}\text { 群落编号 } \\
\text { Community code }\end{array}$ & $\begin{array}{c}\text { 种群 } \\
\text { Population }\end{array}$ & $\begin{array}{l}\text { 株数 } \\
\text { Number }\end{array}$ & $\begin{array}{c}\text { 胸径范围 } \\
D B H \text { Range }(\mathrm{cm})\end{array}$ & $\begin{array}{c}\text { 平均胸径 } \\
\text { Mean } D B H(\mathrm{~cm})\end{array}$ & $\begin{array}{c}\text { 相对多度 } \\
R A\end{array}$ & $\begin{array}{c}\text { 相对频度 } \\
R F\end{array}$ & $\begin{array}{c}\text { 相对显著度 } \\
R D\end{array}$ & $\begin{array}{c}\text { 重要值 } \\
I V\end{array}$ \\
\hline \multirow[t]{6}{*}{ C1 } & 马尾松 Pm & 249 & $3.8 \sim 31.5$ & 18.2 & 27.67 & 11.92 & 92.45 & 132.04 \\
\hline & 三叉苦 El & 115 & $1.1 \sim 4.8$ & 1.7 & 12.78 & 7.62 & 0.36 & 20.75 \\
\hline & 黄牛木 Cl & 65 & $1.1 \sim 4.8$ & 2.6 & 7.22 & 8.94 & 0.50 & 16.67 \\
\hline & 荚口 Vd & 94 & $1.1 \sim 1.9$ & 1.5 & 10.44 & 2.98 & 0.22 & 13.64 \\
\hline & 黄樟 Cp & 37 & $1.1 \sim 12.1$ & 4.6 & 4.11 & 7.28 & 1.10 & 12.49 \\
\hline & 桃金娘 Rt & 56 & $1.1 \sim 3.0$ & 1.4 & 6.22 & 5.30 & 0.12 & 11.64 \\
\hline \multirow[t]{6}{*}{$\mathrm{C} 2$} & 马尾松 Pm & 144 & $1.9 \sim 59.5$ & 14.9 & 10.28 & 5.41 & 55.15 & 70.84 \\
\hline & 鼠刺 Itc & 148 & $1.0 \sim 13.5$ & 4.5 & 10.56 & 5.06 & 4.96 & 20.59 \\
\hline & 桃金娘 Rt & 170 & $1.1 \sim 10.2$ & 1.8 & 12.13 & 4.54 & 1.01 & 17.68 \\
\hline & 木荷 Ss & 50 & $1.1 \sim 50.9$ & 8.6 & 3.57 & 3.84 & 9.14 & 16.54 \\
\hline & 水粟 $\mathrm{Cn}$ & 79 & $1.1 \sim 14.0$ & 3.9 & 5.64 & 5.24 & 2.43 & 13.30 \\
\hline & 黄牛奶树 Sl & 71 & $1.1 \sim 12.2$ & 4.4 & 5.07 & 4.01 & 2.53 & 11.61 \\
\hline \multirow[t]{7}{*}{$\mathrm{C} 3$} & 马尾松 Pm & 52 & $3.8 \sim 50.9$ & 25.6 & 4.49 & 5.45 & 41.96 & 51.90 \\
\hline & 福建青冈 Qc & 136 & $1.2 \sim 30.1$ & 8.3 & 11.73 & 2.72 & 13.53 & 27.99 \\
\hline & 罗浮柿 Dm & 71 & $1.1 \sim 24.5$ & 4.3 & 6.13 & 5.64 & 2.45 & 14.22 \\
\hline & 鼠刺 Itc & 85 & $1.1 \sim 17.5$ & 3.8 & 7.33 & 4.86 & 1.96 & 14.16 \\
\hline & 甜茶椆 Ll & 64 & $1.0 \sim 20.5$ & 5.3 & 5.52 & 3.89 & 3.87 & 13.29 \\
\hline & 水粟 $\mathrm{Cn}$ & 18 & $2.5 \sim 38.5$ & 19.7 & 1.55 & 0.97 & 8.43 & 10.96 \\
\hline & 桃金娘 Rt & 80 & $1.1 \sim 3.0$ & 1.5 & 6.90 & 3.70 & 0.20 & 10.79 \\
\hline \multirow[t]{8}{*}{$\mathrm{C} 4$} & 马尾松 Pm & 18 & $9.2 \sim 61.1$ & 40.7 & 1.00 & 1.87 & 19.25 & 22.12 \\
\hline & 米椎 Cc & 6 & $1.3 \sim 159.2$ & 34.2 & 0.33 & 0.57 & 15.41 & 16.32 \\
\hline & 短花序楠 Mb & 138 & $1.1 \sim 21.5$ & 4.9 & 7.70 & 4.73 & 3.01 & 15.45 \\
\hline & 小叶胭脂 As & 64 & $1.2 \sim 41.1$ & 11.7 & 3.57 & 4.16 & 7.55 & 15.28 \\
\hline & 粘木 Ixc & 48 & $1.1 \sim 39.0$ & 16.9 & 2.68 & 2.30 & 9.37 & 14.35 \\
\hline & 黄枝木 Xh & 143 & $1.3 \sim 11.3$ & 3.6 & 7.98 & 3.73 & 1.39 & 13.10 \\
\hline & 显脉木姜 Np & 127 & $1.1 \sim 12.5$ & 2.2 & 7.09 & 3.30 & 0.56 & 10.95 \\
\hline & 水粟 Cn & 39 & $1.1 \sim 44.2$ & 9.7 & 2.18 & 2.58 & 5.33 & 10.09 \\
\hline \multirow[t]{8}{*}{ C5 } & 黄枝木 Xh & 242 & $1.1 \sim 21.3$ & 5.5 & 17.20 & 5.94 & 5.17 & 28.31 \\
\hline & 水粟 Cn & 32 & $1.3 \sim 84.4$ & 27.1 & 2.27 & 3.14 & 18.16 & 23.57 \\
\hline & 长叶木姜 Le & 102 & $1.2 \sim 38.8$ & 9.9 & 7.25 & 5.28 & 8.22 & 20.75 \\
\hline & 阿丁枫 Ac & 54 & $1.2 \sim 74.8$ & 15.3 & 3.84 & 2.81 & 11.17 & 17.82 \\
\hline & 马尾松 Pm & 7 & $30.9 \sim 74.0$ & 53.3 & 0.50 & 0.83 & 10.89 & 12.22 \\
\hline & 粘木 Ixc & 27 & $1.6 \sim 39.0$ & 19.3 & 1.92 & 2.48 & 5.96 & 10.35 \\
\hline & 小叶胭脂 As & 47 & $1.1 \sim 31.3$ & 8.2 & 3.34 & 3.96 & 2.98 & 10.28 \\
\hline & 红鳞蒲桃 Sh & 76 & $1.1 \sim 20.4$ & 3.4 & 5.40 & 3.96 & 0.69 & 10.05 \\
\hline
\end{tabular}

仅列出各群落中重要值大于 10 的种群 Only those populations with importance value larger than 10 are listed Ac: Altingia chinensis As : Artocarpus styracifolius Cc: Castanopsis carlesii Cn : Castanopsis nigrescens Cp : Cinnamomum porrectum Cl : Cratoxylon ligustrinum Dm : Diospyros morrisiana El : Evodia lepta Itc: Itea chinensis Ixc : Ixonanthes chinensis Ll : Lithocarpus litseifolius Le : Litsea elongate Mb : Machilus breviflora $\mathrm{Np}:$ Neolitsea phanerophlebia Pm : Pinus massoniana Qc:Quercus chungii Rt : Rhodomyrtus tomentosa Ss : Schima superba Sl : Symplocos laurina Sh : Syzygium hancei $\mathrm{Vd}:$ Viburnum dilataum $\mathrm{Xh}:$ Xanthophyllum hainanensis $\quad R A$ : Relative abundance $\quad R F$ : Relative requency $\quad R D:$ Ralative dominance $\quad I V:$ Importance value 
了重要值大于 10 的各种群的有关数据）,马尾松种 群株数、重要值和平均胸径的变动趋势见图 1。

由表 1 及图 1 可以看出, 总体上,马尾松种群的 株数和重要值在群落 $\mathrm{C} 1$ 中最高, 并且按 $\mathrm{C} 1 \rightarrow \mathrm{C} 2 \rightarrow$ $\mathrm{C} 3 \rightarrow \mathrm{C} 4 \rightarrow \mathrm{C} 5$ 的顺序依次递减; 而各群落平均胸径按 $\mathrm{C} 1 \rightarrow \mathrm{C} 2 \rightarrow \mathrm{C} 3 \rightarrow \mathrm{C} 4 \rightarrow \mathrm{C} 5$ 的顺序依次递增 (因为在群落 C2 的一侧出现了一些马尾松幼树, 所以其平均胸径 略低于 C1)。因此, 以空间演替系列代替时间演替 序列, 可以认为 $\mathrm{C} 1 \rightarrow \mathrm{C} 2 \rightarrow \mathrm{C} 3 \rightarrow \mathrm{C} 4 \rightarrow \mathrm{C} 5$ 构成了黑石 顶森林群落演替的一个时间系列。

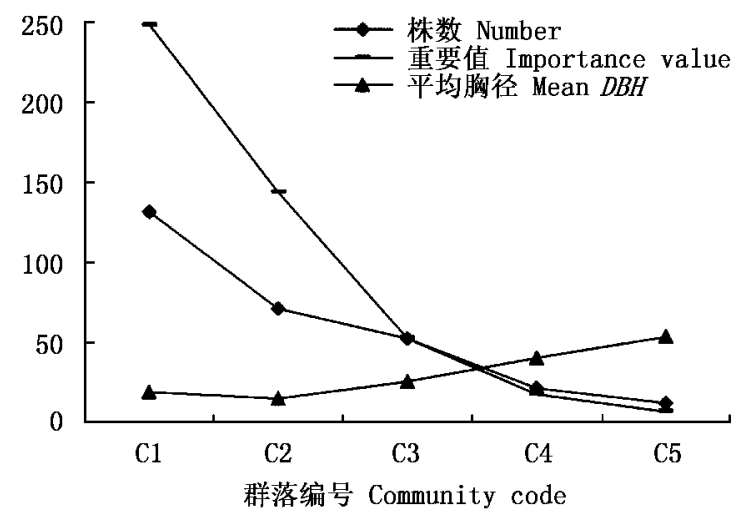

图 1 马尾松种群株数、重要值和平均胸径的变动趋势

Fig. 1 Trend lines of number, importance value and mean $D B H$ of Pinus massoniana population

\section{2 分形分析}

黑石顶 5 个森林群落中重要值大于 10 的种群 的分形维数值及其线性关系存在的尺度范围数据见 表 2 ,马尾松种群计盒维数和信息维数的变动趋势 见图 2。由表 2 及图 2 可以看出, 马尾松种群的计盒 维数和信息维数变动趋势非常接近 均按 $\mathrm{C} 1 \rightarrow \mathrm{C} 2 \rightarrow$ $\mathrm{C} 3 \rightarrow \mathrm{C} 4 \rightarrow \mathrm{C} 5$ 的顺序依次递减。根据马克明和祖元 刚(2000a) 的观点, 可以认为, 马尾松种群在 C1 $\rightarrow$ $\mathrm{C} 2 \rightarrow \mathrm{C} 3 \rightarrow \mathrm{C} 4 \rightarrow \mathrm{C} 5$ 的演替进程中，其空间占据程度、 建群和优势地位均不断下降, 其更新状况逐渐变差。

马尾松种群在群落 C1 中的计盒维数值 (1.782) 和信息维数值 (1.692)均为最高, 表明此阶段中, 其 空间占据程度最高, 其建群和优势地位最明显 其格 局强度的尺度变化最明显, 个体分布的均匀性程度 存在最大差异, 这与其所在群落 C1 的状况是一致 的。实际上, 群落 $\mathrm{C} 1$ 为黑石顶自然保护区最为典型 的马尾松针叶林群落。此群落上层中除有极少量的 八角枫 (Alangium chinense)、酸丰( Choerospondias axillaris) 和山乌柏 (Sapium discolor) 等阳生性阔叶树种 点缀其间外，马尾松在数量上占有绝对优势，其在中 层仍有较大的优势。
马尾松种群在群落 C.5 中的计盒维数值 (0.389) 和信息维数值 $(0.483)$ 均为最低, 表明其空间占据程 度已降至最低, 其在群落中的优势地位已不复存在， 种群格局强度的尺度变化已不明显, 且其更新状况 降至最差。这与其所在群落 C5 的状况也是一致的。

实际上, 在群落 C5 中, 水栗( Castanopsis nigrescens)、长叶木姜 (Litsea elongata) 、阿丁枫( Altingia chinensis) 等中生性阔叶树种已经成为群落上层乔木的 优势树种，而耐荫种类黄枝木 ( Xanthophyllum hainanensis) 在乔木中、下层也占有绝对优势。这 4 个种 群的重要值、计盒维数和信息维数均显著高于马尾 松种群的相应值。

考察马尾松种群的分形维数在其它 3 个群落中 的变动规律, 也会得到相似的结论。因此, 马尾松种 群的分形维数在上述 5 个群落中的变化不但反映了 其种群格局的尺度变化特征及其差异性的数值规 律,也较准确地反映了其更新状况的差异, 具有较明 确的生态学意义，可作为刻划演替系列中种群格局 动态的有效指数。

对比图 1 和图 2 可知,马尾松种群的分形维数 值的变化趋势与其株数和重要值的变化趋势也一 致。结合以上分析, 可以初步断定, 分形分析方法也 可应用于划分森林群落的演替阶段, 其结论不仅与 时空替代分析方法的结论高度一致, 且能够同时反 映种群格局强度的尺度变化规律、在群落中的地位 与作用差异以及更新状况的差异等相关的生态学信 息, 是在缺少动态数据的情况下, 进行森林群落演替 研究的另一可行方法。

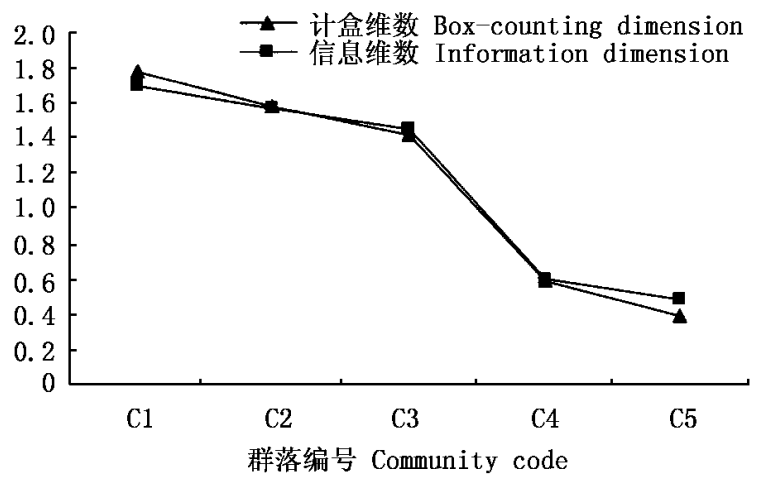

图 2 马尾松种群计盒维数和信息维数的变动趋势

Fig.2 Trend lines of box-counting dimension and information dimension of Pinus massoniana population

\section{3 谱线分析}

为进一步明确演替进程中，马尾松种群重要值、 计盒维数和信息维数在整个群落中的相对变动趋 
表 2 黑石顶自然保护区 5 个森林群落中重要值大于 10 的种群的分形维数

Table 2 Fractal dimensions of populations with importance value larger than 10 in five forest communities in Heishiding Nature Reserve , Guangdong Province

\begin{tabular}{|c|c|c|c|c|c|c|}
\hline \multirow{2}{*}{$\begin{array}{c}\text { 群落编号 } \\
\text { Community code }\end{array}$} & \multirow{2}{*}{$\begin{array}{c}\text { 种群 } \\
\text { Population }\end{array}$} & \multirow{2}{*}{$\begin{array}{c}\text { 尺度范围 } \\
\text { Scale range }(m)\end{array}$} & \multicolumn{2}{|c|}{$\begin{array}{c}\text { 计盒维数 } \\
\text { Box-counting dimension }(\mathrm{Db})\end{array}$} & \multicolumn{2}{|c|}{$\begin{array}{c}\text { 信息维数 } \\
\text { Information dimension (Di) }\end{array}$} \\
\hline & & & $\begin{array}{c}\mathrm{Db} \text { 值 } \\
\mathrm{Db} \text { value }\end{array}$ & $\begin{array}{c}\text { 相关系数 }(R) \\
\text { Correlation coefficient }\end{array}$ & $\begin{array}{c}\text { Di 值 } \\
\text { Di value }\end{array}$ & $\begin{array}{c}\text { 相关系数 } R \\
\text { Correlation coefficient }\end{array}$ \\
\hline \multirow[t]{6}{*}{$\mathrm{C} 1$} & 马尾松 $\mathrm{Pm}$ & $4 \sim 30$ & 1.782 & 0.996 & 1.692 & 0.996 \\
\hline & 三叉苦 El & $4 \sim 30$ & 1.317 & 0.991 & 1.424 & 0.994 \\
\hline & 黄牛木 Cl & $4 \sim 30$ & 1.112 & 0.959 & 1.131 & 0.978 \\
\hline & 荚口 Vd & $4 \sim 30$ & 0.401 & 0.882 & 0.266 & $0.796^{*}$ \\
\hline & 黄樟 Cp & $4 \sim 30$ & 0.933 & 0.943 & 1.021 & 0.950 \\
\hline & 桃金娘 Rt & $4 \sim 30$ & 0.824 & 0.959 & 0.770 & 0.982 \\
\hline \multirow[t]{6}{*}{$\mathrm{C} 2$} & 马尾松 Pm & $4 \sim 30$ & 1.581 & 0.990 & 1.571 & 0.995 \\
\hline & 鼠刺 Itc & $4 \sim 30$ & 1.359 & 0.985 & 1.236 & 0.992 \\
\hline & 桃金娘 Rt & $4 \sim 30$ & 1.259 & 0.979 & 1.097 & 0.972 \\
\hline & 木荷 Ss & $4 \sim 30$ & 0.928 & 0.938 & 0.900 & 0.964 \\
\hline & 水粟 $\mathrm{Cn}$ & $4 \sim 30$ & 1.318 & 0.972 & 1.288 & 0.978 \\
\hline & 黄牛奶树 Sl & $4 \sim 30$ & 1.146 & 0.965 & 1.097 & 0.971 \\
\hline \multirow[t]{7}{*}{$\mathrm{C} 3$} & 马尾松 Pm & $6 \sim 30$ & 1.414 & 0.983 & 1.446 & 0.989 \\
\hline & 福建青冈 Qc & $4 \sim 30$ & 0.924 & 0.956 & 0.898 & 0.955 \\
\hline & 罗浮柿 Dm & $4 \sim 30$ & 1.218 & 0.969 & 1.236 & 0.980 \\
\hline & 鼠刺 Itc & $4 \sim 30$ & 1.115 & 0.969 & 1.081 & 0.976 \\
\hline & 甜茶椆 Ll & $4 \sim 30$ & 0.960 & 0.960 & 0.909 & 0.958 \\
\hline & 水粟 $\mathrm{Cn}$ & $4 \sim 30$ & 0.279 & $0.700^{*}$ & 0.298 & $0.732^{*}$ \\
\hline & 桃金娘 Rt & $4 \sim 30$ & 1.042 & 0.976 & 1.091 & 0.994 \\
\hline \multirow[t]{8}{*}{$\mathrm{CA}$} & 马尾松 Pm & $4 \sim 30$ & 0.586 & 0.907 & 0.598 & 0.924 \\
\hline & 米椎 Cc & $10 \sim 30$ & 0.630 & $0.960^{*}$ & 0.588 & $0.986^{*}$ \\
\hline & 短花序楠 Mb & $4 \sim 30$ & 1.399 & 0.978 & 1.333 & 0.983 \\
\hline & 小叶胭脂 As & $4 \sim 30$ & 1.252 & 0.975 & 1.301 & 0.983 \\
\hline & 粘木 Ixc & $4 \sim 30$ & 0.831 & 0.951 & 0.862 & 0.953 \\
\hline & 黄枝木 Xh & $4 \sim 30$ & 1.415 & 0.993 & 1.426 & 0.996 \\
\hline & 显脉木姜 Np & $4 \sim 30$ & 1.383 & 0.995 & 1.467 & 0.996 \\
\hline & 水粟 Cn & $4 \sim 30$ & 0.844 & 0.953 & 0.863 & 0.954 \\
\hline \multirow[t]{8}{*}{$\mathrm{C} 5$} & 黄枝木 Xh & $4 \sim 30$ & 1.686 & 0.994 & 1.618 & 0.996 \\
\hline & 水粟 Cn & $4 \sim 30$ & 0.728 & 0.905 & 0.780 & 0.923 \\
\hline & 长叶木姜 Le & $4 \sim 30$ & 1.400 & 0.982 & 1.365 & 0.987 \\
\hline & 阿丁枫 Ac & $4 \sim 30$ & 1.000 & 0.975 & 1.074 & 0.984 \\
\hline & 马尾松 Pm & $4 \sim 30$ & 0.389 & 0.878 & 0.483 & 0.893 \\
\hline & 粘木 Ixc & $4 \sim 30$ & 0.644 & 0.910 & 0.666 & 0.942 \\
\hline & 小叶胭脂 As & $4 \sim 30$ & 1.034 & 0.960 & 1.074 & 0.974 \\
\hline & 红鳞蒲桃 Sh & $4 \sim 30$ & 1.166 & 0.975 & 1.188 & 0.987 \\
\hline
\end{tabular}

* 相伴概率 $p$ 值小于 0.01 其余均小于 0.0001 All $p$ values are less than 0.0001 , except those marked with an asterisk $(p<0.01) \quad$ 种名缩写见表 1 Abbreviations of species see Table 1

势，按如下方法绘制上述 3 个数量指标的谱线 :在各 群落内按重要值从大到小排序, 然后以群落编号为 横轴, 分别以各种群的重要值、计盒维数和信息维数 值为纵轴, 生成重要值谱线、计盒维数谱线和信息维 数谱线(图 3)。

由图 3a 重要值谱线可以明显看出, 在森林群落 $\mathrm{C} 1 \rightarrow \mathrm{C} 2 \rightarrow \mathrm{C} 3 \rightarrow \mathrm{C} 4 \rightarrow \mathrm{C} 5$ 的演替进程中，马尾松种群的 重要值不断减小, 重要值谱线的变动幅度不断减小, 并趋于平缓。表明演替由以马尾松为优势种群的单 优群落 (C1) 发展到由多个种群共存的多优群落。
由计盒维数 (图 3b) 和信息维数 (图 3c) 值谱线 可以明显看出, 两种分形维数的变动趋势相似。在 森林群落 $\mathrm{C} 1 \rightarrow \mathrm{C} 2 \rightarrow \mathrm{C} 3 \rightarrow \mathrm{C} 4 \rightarrow \mathrm{C} 5$ 的演替进程中，马 尾松种群的计盒维数和信息维数值不断减小并降至 最低，表明其更新状况渐趋恶化，其空间占据能力不 断下降 种群处于不断衰退状态。而其它各种群, 虽 其重要值谱线平缓, 但其计盒维数和信息维数谱线 却出现不同程度的波动, 这是种群空间占据能力和 更新状况存在差异的反映。如在群落 C.5 中, 虽然黄 枝木的重要值 (28.31) 与水栗的重要值 (23.57) 相 
近, 但黄枝木 (计盒维数 1.689 , 信息维数 1.618) 属 而荫种类 其空间占据能力和更新能力均要强于属 中生性种类的水栗 (计盒维数 0.728 , 信息维数 0.780)。因此,前者在乔木中、下层均占有绝对优 势,而后者成为上层乔木中的优势树种。
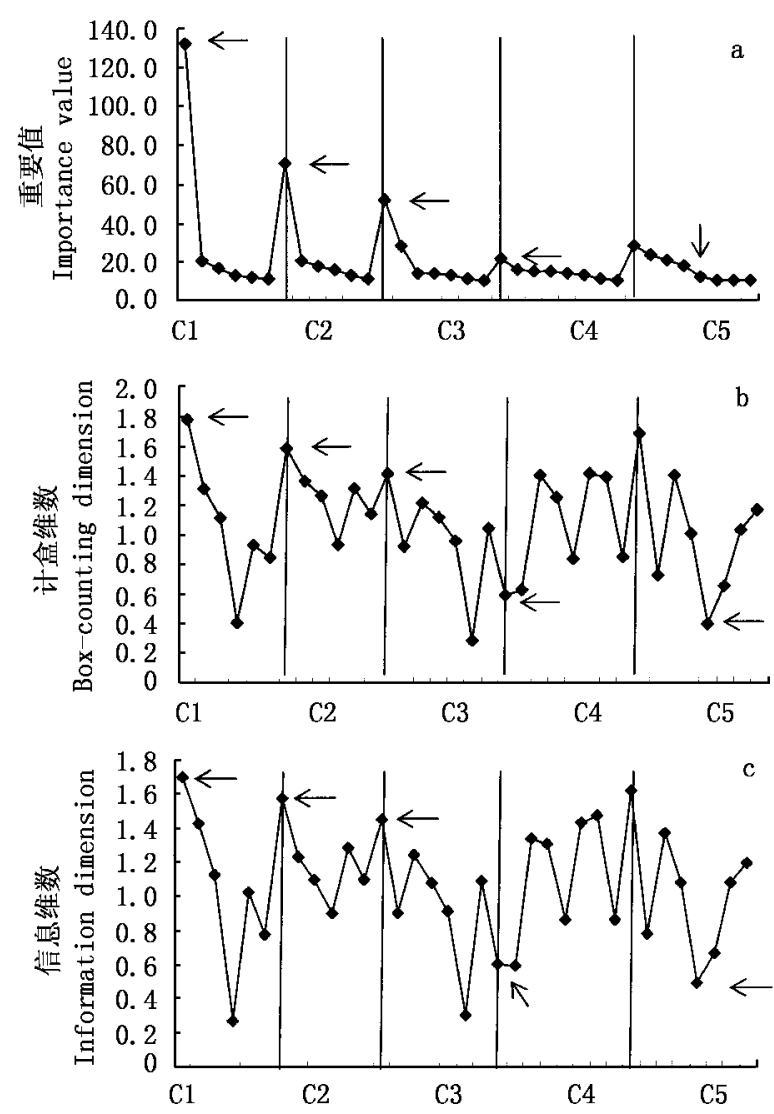

图 3 黑石顶自然保护区 5 个森林群落的重要值 (a)、 计盒维数 (b) 和信息维数 (c) 谱线

Fig.3 Spectral lines of importance values(a), box-counting dimensions (b) and information dimensions $(\mathrm{c})$ for five forest communities in Heishiding Nature Reserve, Guangdong Province

箭头所指符号代表马尾松种群 Symbol pointed with an arrow is for Pinus massoniana population

\section{4 讨论与结论}

演替是生态系统最常见的自然现象之一。演替 理论是合理经营、有效利用自然资源以及改造、恢复 与重建退化生态系统的重要理论基础。一般认为, 群落演替 (Community succession), 或称为生态演替 (Ecological succession), 是指群落经过一定历史发展 时期, 由一种类型转变为另一种类型的顺序过程, 也 就是在一定区域内群落的发展替代过程 (彭少麟, 1996)。由于群落演替是一个复杂的渐变过程, 同时 受到多种因素的共同影响, 而且其研究一般要涉及 到时间因子，因此，通过固定样地获得时间序列上的
数据资料进行演替研究是理论上最理想的方法。然 而,由于植物群落特别是森林群落的演替跨越时间 尺度太大，这种获取时间序列数据的方法在实践上 存在很大的困难，因此实际采用的方法大多是时空 替代方法, 即按一定规则以样地的空间序列组成其 时间序列, 并据此进行生态系统的演替研究。虽然 应用这种方法可能会产生较大的误差, 但在没有其 它可行的方法替代时，仍一直被沿用至今 (江洪等, 2003)。

本文根据时空替代原则，利用马尾松种群的重 要值-平均胸径的变动趋势, 确定了群落演替的时间 系列 ;并在此基础上 求算马尾松种群的计盒维数和 信息维数两种分形维数。结果显示, 马尾松种群的 计合维数和信息维数 2 个参数值均呈递减趋势, 与 其个体数、重要值 2 个参数值的变动趋势一致。分 形分析与重要值-平均胸径分析的结果一致，表明在 本研究中使用 空间代替时间” 原则是合适的。所研 究的 5 个森林群落按 $\mathrm{C} 1 \rightarrow \mathrm{C} 2 \rightarrow \mathrm{C} 3 \rightarrow \mathrm{C} 4 \rightarrow \mathrm{C} 5$ 的顺序 构成了黑石顶森林群落演替的一个时间系列。马尾 松种群的平均胸径呈增长趋势, 其重要值、计盒维数 和信息维数均呈下降趋势, 表明伴随着演替过程, 马 尾松种群的个体不断生长, 但因更新状况渐趋恶化, 其空间占据能力不断下降, 种群呈衰退趋势。随着 马尾松种群在群落中的建群及优势地位逐渐被其它 种群取代 群落将由以马尾松占绝对优势的单优群 落演替为多优常绿阔叶林群落。

生态系统是复杂的非线性体系，分形理论是对 其层次性和复杂性进行简明数量描述的重要手段 (Sugihara \& May ,1990; 辛晓平等,2000)。例如在存 在自相似性的尺度范围内, 分形维数可准确地定量 描述群落的 $\alpha$ 多样性(王永繁等, 2002)。植被是一 个分形体，其在所有尺度上都有细节 (Palmer ,1988)， 植被格局在各个层次上都具有分形特征,可以运用 不同的分形方法进行描述 (马克明和祖元刚, 2000b)。如梁士楚和王伯荪 (2002) 研究了木榄 (Bruguiera gymnorrhiza) 种群高度结构的分形特征； 余世孝和郭泺 (2005)对泰山风景区的景观特性及其 变化的分形性质进行了研究，结果表明分维值的大 小反映了景观斑块面积、数量和平均班块面积等因 素的相互作用。

虽然分形理论在生态学中的应用研究进展很 快, 但主要集中在生态学格局特征和空间尺度转换 分析、生态空间异质性研究、种群和群落性状的时间 序列分析等方面(辛晓平等，2000），且总体上还处于 
比较零散、不系统的阶段(马克明和祖元刚，2000b)。 由于长期的格局动态数据难以获取, 应用分形方法 揭示植被格局动态与生态学过程关系的研究还比较 少(辛晓平等,2000)。本文利用分形方法研究植被 演替过程中种群空间格局动态变化的分形特征是一 次有益的尝试。研究结果表明,分形分析是群落演 替过程中种群格局动态研究的有效方法。

\section{参 考 文 献}

DaiXH (戴小华), Yu SX (余世孝), Lian JY (练琚 $\square)$ (2003). Interspecific segregation in a tropical rain forest at Bawangling Nature Reserve, Hainan Island. Acta Phytoecologica Sinica (植物生态学报), 27, 380 - 387. (in Chinese with English abstract)

Ding SY (丁圣彦), Song YC (宋永昌) (1998). Declining causes of Pinus massoniana in the processes of succession of evergreen broad-leaved forest. Acta Botanica Sinica (植物学报), 40, 755 - 760. (in Chinese with English abstract)

Fang W (方炜), Peng SL (彭少麟) (1995). Changes of tree species in the succession process of Pinus massoniana community in Dinghushan, Guangdong, P. R. China. Journal of Tropical and Subtropical Botany (热带亚热带植物学报)，3，30-37.

(in Chinese with English abstract)

Jiang H (江洪), Zhang YL (张艳丽), Strittholt JR (2003). Spatial analysis of disturbances and ecosystem succession. Acta Ecologica Sinica (生态学报), 23, 1861 - 1876. (in Chinese with English abstract)

Liang SC (梁士楚), Wang BS (王伯荪) (2002). Fractal characteristics of the height structure of Bruguira gynmorrhiza population. Acta Phytoecologica Sinica (植物生态学报), 26, 408 412. (in Chinese with English abstract)

Ma KM (马克明), Zu YG (祖元刚) (2000a). Fractal properties of the spatial pattern of Larix gmelini population-information dimension. Acta Ecologica Sinica (生态学报), 20, 187 - 192. (in Chinese with English abstract)

Ma KM (马克明), Zu YG (祖元刚) ( 2000b). Fractal properties of vegetation pattern. Acta Phytoecologica Sinica (植物生态学 报), 24, 111-117. (in Chinese with English abstract)

Palmer MW (1988). Fractal geometry: a tool for describing spatial patterns of plant communities. Vegetatio, 75, 91-102.

Peng SL (彭少麟) (1996). The Dynamics of Community in Southern Subtropical Forest (南亚热带植物群落动态学). Science Press, Beijing. (in Chinese)

Sugihara G, May RM (1990). Application of fractals in ecology . Trends in Ecology and Evolution, 5, 79 - 86.

Wang BS (王伯荪), Ma MJ (马曼杰) (1982). Succession of forest communities in Dinghushan Natural Reserve. Tropical and
Subtropical Forest Ecosystem (热带亚热带森林生态系统研 究), 1, 142 - 156. (in Chinese with English abstract)

Wang BY (王本洋), Yu SX (余世孝) (2003). Technique for the development of data dictionary based on ArcView GIS. Remote Sensing Technology and Application (遥感技术与应用), 18, 429-433. (in Chinese with English abstract)

Wang BY (王本洋), Yu SX (余世孝) (2005). Multi-scale analyses of population distribution patterns. Acta Phytoecologica Sini$c a$ (植物生态学报), 29, 235-241. (in Chinese with English abstract)

Wang YF (王永繁), Yu SX (余世孝), Liu WQ (刘蔚秋) (2002). A new species diversity index and its fractal analysis. Acta Phytoecologica Sinica (植物生态学报), 26, 391 - 395. (in Chinese with English abstract)

Xin XP (辛晓平), Xu B (徐斌), Shan BQ (单保庆), Li JD (李建东), Yang YF (杨允菲) (2000). Patch dynamics and scale-transition analysis of grassland in restoration succession. Acta Ecologica Sinica (生态学报), 20, 587 - 593. (in Chinese with English abstract)

Yang QP (杨清培), Li MG (李鸣光), Li RW (李仁伟) (2001). Studies on the dynamic succession of Pinus massoniana community in Heishiding Natural Reserve. Guihaia (广西植 物 ), 21, 295-299. (in Chinese with English abstract)

Yu SX (余世孝), Guo L (郭泺) (2005). Landscape structures and fractal analysis of Taishan Mountain, Shandong Province. Acta Ecologica Sinica (生态学报), 25, 129 - 134. (in Chinese with English abstract)

Yu SX (余世孝), Li Y (李勇), Wang YF (王永繁), Zhou CF (周灿芳) (2000). The vegetation classification and its digitized map of Heishiding Nature Reserve, Guangdong. I . The distribution of the vegetation type and formation. Acta Scientiarum Naturalium Universitatis Sunyatseni (中山大学学报 (自然科学 版) ), 39,61-66. (in Chinese with English abstract)

Yu SX (余世孝), Zang RG (藏润国), Jiang YX (蒋有绪) (2001). Spatial analysis of species diversity in the tropical vegetation along the vertical belt at Bawangling Nature Reserve, Hainan Island. Acta Ecologica Sinica (生态学报), 21, 1438 1443. (in Chinese with English abstract)

Yu SX (余世孝), Zong GW (宗国威), Chen ZY (陈兆莹), Zang RG (留润国), Yang YC(杨彦承) (1998). Comparison of ecological entropy with random and systematic sampling. Acta Phytoecologica Sinica (植物生态学报), 22, 473 - 480.（in Chinese with English abstract)

Zan QJ (备启杰), Li MG (李鸣光), Wang BS (王伯荪), Zhou $\mathrm{XY}$ (周先叶) (2000). Dynamics of community structure in successional process of needle and broad-leaved forest in Heishiding of Guangdong. Chinese Journal of Applied Ecology (应用生态学 报), 11, 1-4. (in Chinese with English abstract) 